$$
\begin{aligned}
& \text { NCC2-3TI } \\
& \text { IN-23-CR }
\end{aligned}
$$

\title{
Theoretical study of the C-H bond dissociation energy of acetylene
}

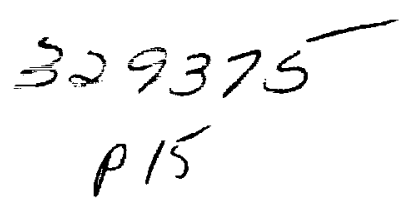

Charles W. Bauschlicher, Jr. and Stephen R. Langhoff

NASA Ames Research Center

Moffett Field, CA 94035

Peter R. Taylor

ELORET Institute $\dagger$

Sunnyvale, CA 94087

Abstract

We present a theoretical study of the convergence of the C-H bond dissociation energy $\left(D_{0}\right)$ of acetylene with respect to both the one- and $n$-particle spaces. Our best estimate for $D_{0}$ of $130.1 \pm 1.0 \mathrm{kcal} / \mathrm{mole}$ is slightly below previous theoretical estimates, but substantially above the value determined using Stark anticrossing spectroscopy that is asserted to be an upper bound.

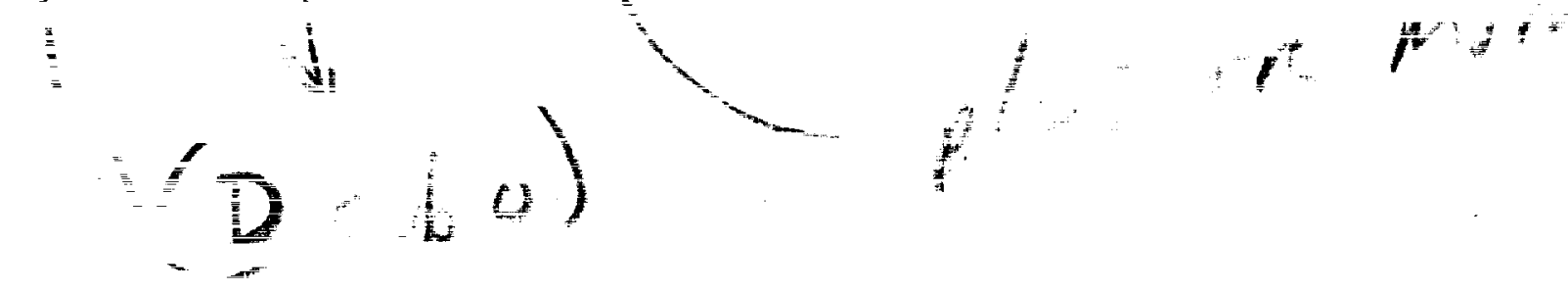

$$
\begin{aligned}
& \text { (NASA-CR-187850) THEOREIICAL STUDY UF THE } \\
& \text { C-H BOND DISSICIATION ENERGY OF ACETYLENE } \\
& \text { (ElORET CORP.) } 15 \mathrm{p} \\
& \text { CSCL OTA }
\end{aligned}
$$$$
\text { N91-15055 }
$$$$
\text { Unclis }
$$$$
63 / 23 \quad 0329375
$$

$†$ Mailing address: NASA Ames Research Center, Moffett Field, CA 94035 


\section{Introduction}

There is currently considerable controversy regarding the $\mathrm{C}-\mathrm{H}$ dissociation energy of acetylene, $D_{0}(\mathrm{HCC}-\mathrm{H})$. This is in large part due to the low and presumably very accurate value determined by Green, Kinsey, and Field (GKF) [1] using Stark anticrossing spectroscopy. Their result, $126.647(2) \mathrm{kcal} / \mathrm{mole}$, is claimed to be an upper bound, and is consistent with the $127 \pm 1.5 \mathrm{kcal} / \mathrm{mole}$ estimate of Segall et al. [2] obtained by measuring the kinetic energy (K.E.) of the hydrogen atom fragment using Doppler multiphoton ionization spectroscopy. However, these values are substantially less than previous theoretical estimates $[3,4]$ and other recent experimental results, such as the $D_{0}$ value of $131.3 \pm 0.7 \mathrm{kcal} /$ mole measured by Ervin et al. [5] using the techniques of negative ion photoelectron spectroscopy and gas phase proton transfer kinetics.

In this Letter we present a theoretical study of the C-H dissociation energy of acetylene, with special emphasis on convergence with improvements in the oneand $n$-particle basis sets. We examine the $n$-particle space convergence by performing multireference CI (MRCI) calculations with reference configurations selected at different thresholds from a complete-active-space SCF (CASSCF) wave function. The effect of higher than double excitations is accounted for using either a Davidson correction [6] or the averaged coupled-pair functional method (ACPF) [7]. In fact, since the MRCI technique can treat $\mathrm{C}_{2} \mathrm{H}_{2}$ and $\mathrm{C}_{2} \mathrm{H}$ to equal accuracy, our results are relatively insensitive to correction for higher excitations. We also compare two single-reference treatments, the coupled-pair functional (CPF) method [8] and its modified form (MCPF) [9], with MRCI. As both CPF and MCPF reproduce the MRCI results fairly well, we have performed most of our one-particle basis set convergence studies with these less expensive methods. By employing a well-defined sequence of basis sets of increasing size we are able to estimate the basis set limit dissociation energy with considerable confidence.

Our best estimate of the dissociation energy $\left(D_{e}\right)$, when combined with computed or experimental zero-point corrections, indicates that $D_{0}$ is $130.1 \pm 1.0 \mathrm{kcal} / \mathrm{mole}$ with a high degree of certainty.

II. Methods

The one-particle basis sets employed for carbon in this study are constructed using general contractions based on atomic natural orbitals (ANOs) [10]. 
We have used two different contractions, [ $\left.\begin{array}{lllll}4 s & 3 p & 2 d & 1 f\end{array}\right]$ and [ [ $\left.\begin{array}{lllll}5 s & 4 p & 3 d & 2 f & 1 g\end{array}\right]$, of the $(13 s 8 p 6 d 4 f 2 g)$ primitive Gaussian set. The $s$ and $p$ exponents are taken from van Duijneveldt [11] and the polarization functions are given in Ref. 12. For hydrogen, we have used the $8 \mathrm{~s}$ primitive set optimized by van Duijneveldt [11], augmented with a $(6 p 4 d)$ polarization set [10]. Three different ANO contractions were considered for hydrogen, namely [3s $2 p 1 d]$, $\left[\begin{array}{lll}4 s & 2 p & 1 d\end{array}\right]$, and [ $\left.\begin{array}{lll}4 s & 3 p & 2 d\end{array}\right]$.

To obtain a balanced treatment of $\mathrm{C}_{2} \mathrm{H}_{2}$ and $\mathrm{C}_{2} \mathrm{H}$, we have used MRCI and ACPF treatments based on CASSCF wave functions. To minimize size-consistency problems, the $D_{e}$ value was computed using a supermolecule approach. The CASSCF active space included the $\mathrm{C} 2 s$ and $2 p$ and the $\mathrm{H} 1 s$ electrons and orbitals. In $C_{2 v}$ symmetry this corresponds to six $a_{1}$, two $b_{1}$, and two $b_{2}$ orbitals, and generates 5180 configuration-state functions (CSFs).

All single and double replacements from the CASSCF configuration space would result in prohibitively long CSF expansions, so it was necessary to select occupations based on their importance in the CASSCF wave function. The reference lists for the MRCI and ACPF treatments included all occupations for which the absolute value of the coefficient of any one of the component spin couplings exceeded a designated threshold in the CASSCF wave function, either at the equilibrium or dissociated (supermolecule) geometry. Selection thresholds of 0.05 and 0.025 were employed, resulting in 14 and 39 reference occupations, respectively. Using the latter reference space, the MRCI treatment (denoted MRCI(0.025)) resulted in a CSF expansion exceeding 2.5 million CSFs in the $[4 s 3 p 2 d 1 f / 4 s 2 p 1 d]$ basis, so it was not feasible to consider smaller selection thresholds. However, as we show below, the equilibrium and dissociated geometries are equally well described at the MRCI(0.025) level, so it is unlikely that the MRCI(0.025) dissociation energy will differ significantly from the value that would be obtained either with smaller selection thresholds or with the entire CASSCF configuration space as references. The effect of higher than double excitations was estimated using both the multireference analog of the Davidson correction (denoted $+Q$ ) [6] and the ACPF approach [7]. Correlation effects involving the core ( $\mathrm{C} 1 s$ ) electrons were excluded from consideration, but this is expected to affect $D_{0}$ by less than $0.2 \mathrm{kcal} / \mathrm{mole}$ [13].

Since the MRCI $D_{0}$ values were well reproduced by the much less computationally intensive CPF and MCPF methods, basis set calibration studies were performed using these single-reference-based approaches, following SCF calculations 
with symmetry and equivalence restrictions imposed. The first-order interacting space restriction is also imposed in the CPF and MCPF calculations. The $D_{e}$ value is computed as the energy difference between the equilibrium geometry of $\mathrm{C}_{2} \mathrm{H}_{2}$ and the energy for the ${ }^{3} \Sigma^{+}$state with one hydrogen removed to $100 a_{0}$.

Zero-point vibrational energy contributions to $D_{0}$ were computed using

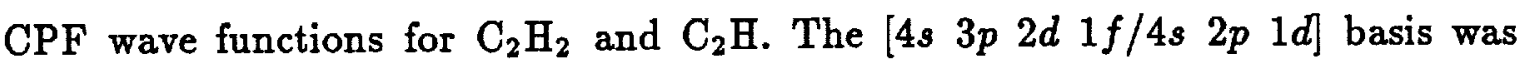
used. Harmonic frequencies were obtained from force constants computed by finite difference methods.

The CASSCF/MRCI and SCF/CPF calculations were performed using the MOLECULE-SWEDEN [14] program system. The calculations were performed on the NASA Ames Central Computer Facility and NAS facility CRAY Y-MP/832 computers.

III. Results and discussion

To determine a definitive $\mathrm{C}-\mathrm{H}$ bond dissociation energy for acetylene, we must demonstrate convergence in both the one- and $n$-particle spaces. Other errors such as invoking the Born-Oppenheimer approximation, neglect of correlation involving the $\mathrm{C} 1 s$ electrons, and relativistic effects are expected to contribute at most a few tenths of a kcal $/$ mole to $D_{e}$. Ideally, we wish to carry out the study of the convergence of the one-particle basis at a high level of correlation treatment so that effects arising from possible coupling between the one and $n$-particle treatments are minimized. The single-reference-based approaches yield $D_{e}$ values that are within 1.0 (CPF) or 0.7 (MCPF) kcal/mole of our most accurate multireference-based correlation approaches in the $[4 s 3 p 2 d 1 f / 4 s 2 p 1 d]$ contracted basis. This relatively small error combined with the good agreement between the CPF and MCPF approaches indicates that the CPF method should give a reliable estimate of the effect of basis set saturation.

The results of our basis set saturation study are summarized in Table I. The $D_{e}$ values are computed based on geometries optimized at the $\mathrm{MRCI}(0.05)$ level. Only the CPF results are given, since the MCPF dissociation energies are uniformly $0.3 \mathrm{kcal} / \mathrm{mole}$ less. The $D_{e}$ values generally increase with basis set improvement; an exception occurs on going from the [4s $3 p 2 d 1 f / 3 s 2 p 1 d]$ to the

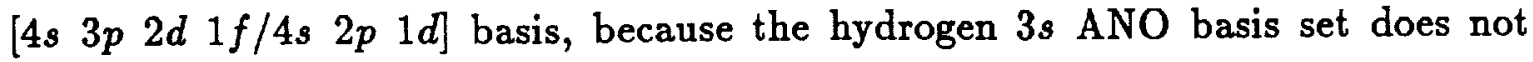
accurately describe the $\mathrm{H}$ atom. This shortcoming is remedied with the $4 s$ basis, 
which reproduces the hydrogen atom energy to within $0.2 \mathrm{kcal} / \mathrm{mole}$. Adding an additional $s$ through $f$ ANO function to carbon and an additional $p$ and $d$ function to hydrogen increases $D_{e}$ by only $0.1 \mathrm{kcal} / \mathrm{mole}$, while adding a $g$ function to carbon increases it by an additional $0.3 \mathrm{kcal} /$ mole. Thus the [4s $3 p 2 d 1 f / 3 s 2 p 1 d]$ basis obtains a fortuitiously good $D_{e}$ value, because the limitations in the $\mathrm{H} s$ basis approximately cancel the effect of improving the polarization basis. Additional basis set improvements are likely to further increase $D_{e}$ slightly $(0.1-0.3 \mathrm{kcal} / \mathrm{mole})$, because the correlation energy of $\mathrm{C}_{2} \mathrm{H}_{2}$ is larger than that of $\mathrm{C}_{2} \mathrm{H}$, and is expected to converge more slowly with the one-particle basis. This claim cannot be made with the same confidence as would be the case for a diatomic dissociation, however, because of the more complicated correlation effects that arise in molecular, as opposed to atomic, fragments.

Some insight into the $n$-particle requirements can be obtained from an analysis of the work of Curtiss and Pople [3] based on the G1 approach [15], in which electron correlation is handled initially by performing fourth-order MøllerPlesset perturbation theory (MP4) calculations. A correction is then added from quadratic CI (QCI) calculations [16]. For the C-H bond dissociation energy of acetylene, the QCI correction decreases the dissociation energy by a large amount $(8.3 \mathrm{kcal} / \mathrm{mole})$. This is a consequence of the fact that a single-reference description of the closed shell ${ }^{1} \Sigma_{g}^{+}$ground state of $\mathrm{C}_{2} \mathrm{H}_{2}$ is superior to that of the ${ }^{2} \Sigma^{+}$ground state of the $\mathrm{C}_{2} \mathrm{H}$ radical. This suggests that single-reference-based techniques may overestimate the binding energy if infinite-order effects and the contributions of higher excitations are not fully accounted for.

CASSCF wave functions supply a more equivalent description of $\mathrm{C}_{2} \mathrm{H}_{2}$ and $\mathrm{C}_{2} \mathrm{H}$ than SCF wave functions, and thus differential correlation effects are easier to account for using MRCI wave functions. Such CASSCF/MRCI calculations have been found to perform very well in relation to full CI (FCI) benchmark calculations [17], but these have been limited to dissociation to atomic fragments, where the dissociation products were always easier to describe than the molecular system. Since we are unable to perform FCI benchmark calculations for acetylene in double zeta plus polarization (DZP) or better quality basis sets, we have used both the $+Q$ correction and the more rigorous ACPF method to provide independent estimates of the effect of higher excitations.

The $D_{e}$ values computed using the $\left[\begin{array}{llllll}4 s & 3 p & 2 d & 1 f / 3 s & 2 p & 1 d\end{array}\right]$ and 


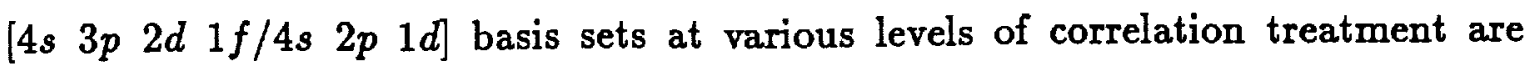
summarized in Table II. We note again that the $D_{e}$ values are decreased by about $0.5 \mathrm{kcal} / \mathrm{mole}$ if the $3 \mathrm{~s}$ hydrogen basis is replaced by the $4 s$ basis, because of the error in describing $H$ atom in the former. Our results demonstrate that the effect of improving the $\mathrm{H}$ basis is essentially independent of the level of correlation treatment, thereby justifying our use of CPF wave functions in our basis set study above.

The MRCI+Q and ACPF $D_{e}$ values are in good agreement at both selection thresholds, but the effect of higher excitations on $D_{e}$ changes sign as the selection threshold is tightened. This results from the fact that at a threshold of 0.05 the reference configurations provide a better description of $\mathrm{C}_{2} \mathrm{H}_{2}$ at equilibrium than at dissociation: the weight of the reference configurations in the MRCI wave function is greater at equilibrium. As a consequence, $D_{e}$ computed using the reference energies is $6 \mathrm{kcal} / \mathrm{mole}$ larger than if the CASSCF energies are used. The bias in the reference level description leads to an artificially large correlation energy (defined here as the difference between the MRCI and reference energies) in the dissociated molecule and hence to an overestimated $+Q$ correction that reduces $D_{e}$. At the 0.025 threshold, the reference space comprises a nearly identical fraction of the MRCI wave function at the equilibrium and at dissociation, and $D_{e}$ computed using the reference energies is only $0.6 \mathrm{kcal} /$ mole smaller than the CASSCF value. The 0.025 threshold thus treats equilibrium and dissociation equivalently, resulting in a $+Q$ correction that increases $D_{e}$. In addition, since $D_{e}$ computed from the reference energies is too small, any bias at the 0.025 threshold level would be expected to produce an MRCI $D_{e}$ value that is too small. We believe that the MRCI(0.025) $D_{e}$ value of $137.27 \mathrm{kcal} / \mathrm{mole}$ should be a lower bound, as higher excitations certainly increase $D_{e}$. This is also supported by the good agreement between the ACPF and MRCI $+Q$ calculations. We consider the most accurate $D_{e}$ values to be those at the $\operatorname{ACPF}(0.025)$ level, as they should be near the $n$-particle limit.

One effect that can artificially increase $D_{e}$ is basis set superposition error (BSSE). We have computed the BSSE at the $\mathrm{C}_{2} \mathrm{H}$ equilibrium geometry using the couterpoise method. At the CPF level the BSSE correction is 0.75 and $0.24 \mathrm{kcal} / \mathrm{mole}$ in the $[4 s 3 p 2 d 1 f / 4 s \quad 2 p 1 d]$ and [ $5 s \quad 4 p 3 d 2 f \quad 1 g / 4 s 3 p 2 d]$ basis sets, respectively. Thus the relatively small apparent increase in $D_{e}$ with basis set improvement results from a partial cancellation between the reduction in BSSE and 
the true increase in $D_{e}$. Our correction of $0.2 \mathrm{kcal} / \mathrm{mole}$ for further basis set saturation is consistent with observed relationships between basis set incompleteness and BSSE when the basis set is nearly complete. We should also note that use of an isogyric correction would increase $D_{e}$ by only $0.17 \mathrm{kcal} / \mathrm{mole}$ - our computed error in the dissociation energy of $\mathrm{H}_{2}$.

To obtain our best estimate for $D_{e}$, we must correct the $\operatorname{ACPF}(0.025)$ values for basis set incompleteness. We add $0.4 \mathrm{kcal} / \mathrm{mole}$ for improving the oneparticle basis from [ $4 s 3 p 2 d 1 f / 4 s 2 p 1 d$ ] to [ $5 s 4 p 3 d 2 f 1 g / 4 s 3 p 2 d$ ], based on the CPF results of Table I. We further increase our value by $0.2 \mathrm{kcal} / \mathrm{mole}$ to account for the addition of $h$ and higher angular momentum functions and saturation of the $l \leq 5$ shells on carbon, as well as $f$ functions and saturation of the $l \leq 2$ shells for hydrogen. Therefore our best estimate for $D_{e}$ is about $138.2 \mathrm{kcal} / \mathrm{mole}$. To this we assign an uncertainty of $0.7 \mathrm{kcal} / \mathrm{mole}$ to encompass the value before correction for basis set incompleteness; this error is of the same magnitude as the error in the bond dissociation energy of $\mathrm{CH}$ radical [12].

In order to compare our results with the experimentally determined $D_{0}$ values, we must correct our computed $D_{e}$ values for zero-point energy. Vibrational energies for both $\mathrm{C}_{2} \mathrm{H}_{2}$ and $\mathrm{C}_{2} \mathrm{H}$ are compared with experiment [18-20] in Table III. The CPF method gives harmonic frequencies for $\mathrm{C}_{2} \mathrm{H}_{2}$ that agree well with experiment [18]. The maximum error is only $47 \mathrm{~cm}^{-1}$ (for the symmetric CH stretch) and the error in the zero-point energy is $0.15 \mathrm{kcal} / \mathrm{mole}$. However, the zero-point energy of $\mathrm{C}_{2} \mathrm{H}$ is less easy to estimate reliably. The $\nu_{3}$ band ( $\mathrm{CH}$ fundamental) has not been conclusively assigned from experiment, and the original assignment [21] of this band as $3612 \mathrm{~cm}^{-1}$ based on isotopic studies of infrared absorptions is almost certainly incorrect. Not only is it in substantial disagreement with our CPF value $\left(3328 \mathrm{~cm}^{-1}\right)$, but if it is used in an empirical force constant/bond length correlation formula [21] it leads to a ridiculously short $\mathrm{C}-\mathrm{H}$ bond length of $1.915 a_{0}$. Our computed frequency gives a bond length estimate of $1.996 a_{0}$ using the same formula, in much better agreement with the actual CPF value of $2.016 a_{0}$. Hence we prefer our computed value for the $\mathrm{CH}$ stretch. (Note, however, that a larger value, $3550 \mathrm{~cm}^{-1}$, is obtained in the $a b$ initio study of Peric et al. [22].) The C-C stretch and bending fundamentals are even more difficult to compute accurately, owing to the strong interaction between these two modes induced by the avoided crossing of the lowest ${ }^{2} A^{\prime}$ state with the ${ }^{2} A^{\prime}$ component of the ${ }^{2} \Pi$ state near its minimum at a $\mathrm{C}-\mathrm{C}$ bond 
length of $2.5 a_{0}$. For the purpose of computing the zero-point correction we have used the experimental $\nu_{2}$ value [19] of $371.6 \mathrm{~cm}^{-1}$ and have considered both the experimental [20] and CPF values for $\nu_{1}$.

The zero-point energy for $\mathrm{C}_{2} \mathrm{H}$ is 8.45 and $8.70 \mathrm{kcal} / \mathrm{mole}$ using $\left(\nu_{1}, \nu_{2}, \nu_{3}\right)$ values $\left(\mathrm{cm}^{-1}\right)$ of $(1840,371,3328)$ and $(2017,371,3328)$ for $\mathrm{C}_{2} \mathrm{H}$, respectively. Combining these with the experimental zero-point energy of $16.68 \mathrm{kcal} / \mathrm{mole}$ for $\mathrm{C}_{2} \mathrm{H}_{2}$ leads to a total zero-point correction of 8.23 and $7.98 \mathrm{kcal} / \mathrm{mole}$. These values are similar to the value of $8.05 \mathrm{kcal} / \mathrm{mole}$ used by Curtiss and Pople [3]. Since it is highly unlikely that the zero-point energy of $\mathrm{C}_{2} \mathrm{H}$ could be less than the first set of values, $8.23 \mathrm{kcal} /$ mole must be considered to be an upper bound to the zero-point correction. Combining these zero-point corrections with our best estimate of $138.2 \mathrm{kcal} / \mathrm{mole}$ for $D_{e}$ leads to $D_{0}$ values of 130.0 and $130.2 \mathrm{kcal} / \mathrm{mole}$. We thus take our best estimate of $D_{0}$ to be $130.1 \mathrm{kcal} / \mathrm{mole}$, and add an additional $0.3 \mathrm{kcal} / \mathrm{mole}$ to our error bars for uncertainties in the zero-point correction. We feel that our estimate of $130.1 \pm 1.0 \mathrm{kcal} / \mathrm{mole}$ has a $90 \%$ probability of encompassing the true value. In view of our expectation that we will approach $D_{0}$ from below it seems likely that the actual value will lie in the upper half of our estimated uncertainty.

Our theoretical estimate of $130.1 \pm 1.0 \mathrm{kcal} / \mathrm{mole}$ is compared with other recent experimental $[1,2,5,23,24]$ and theoretical $[3,4,25]$ determinations in Table IV. The agreement between the theoretical calculations is reasonably good, especially considering that the $D_{0}$ value of Curtiss and Pople [3] is likely to be slightly too high as a result of underestimating the effects of electron correlation in $\mathrm{C}_{2} \mathrm{H}$ and favoring $\mathrm{C}_{2} \mathrm{H}_{2}$ somewhat. The calculations of Montgomery and Petersson [4] were performed at the QCI level; they include extrapolation to an estimated complete basis set limit. Their result is a little higher than ours: it is tempting to ascribe this again to the tendency of single-reference-based methods to favor $\mathrm{C}_{2} \mathrm{H}_{2}$. In this regard we note that the QCISD(T) energy for $\mathrm{C}_{2} \mathrm{H}$ is $1.39 \mathrm{kcal} / \mathrm{mole}$ above the FCI energy in an STO-3G basis [26], so the RMS error bars in Ref. 4, determined from atomization energies for simpler systems, may be too optimistic for acetylene. Montgomery and Petersson obtain an excellent electron affinity (EA) for $\mathrm{C}_{2} \mathrm{H}$, which would suggest that their correlation treatment is not biased towards the closed-shell system, although this could result from a cancellation of errors that may not occur in the calculation of the dissociation energy. On balance, we conclude that our results and those of Montgomery and Petersson are essentially in agreement. Very recently, 
Wu and Carter [25] have obtained a $D_{0}$ value of $129.7 \mathrm{kcal} / \mathrm{mole}$ using an MRCI treatment in a DZP basis and a zero-point correction derived from experimental harmonic frequencies. Their result is very similar to ours, especially considering that basis set improvements will probably increase their value.

The agreement between our value and recent experimental measurements is also satisfactory, excluding the recent experiment interpreted as providing an upper bound of $126.647 \mathrm{kcal} / \mathrm{mole}$ by GKF [1]. The validity of their upper bound rests on the contention that observed increases in molecular decay rates are due to predissociation rather than any other Stark-induced nonradiative or radiative phenomenon. Although these authors carefully consider various alternatives, it still seems likely that another explanation exists, considering the wealth of data supporting a $D_{0}$ value of greater than $129.0 \mathrm{kcal} /$ mole.

\section{Conclusions}

We have presented a systematic study of the C-H bond dissociation energy in acetylene with respect to improvements in both the one- and $n$-particle treatments. Our best estimate for the $D_{0}$ value of $130.1 \pm 1.0 \mathrm{kcal} / \mathrm{mole}$ is in good agreement with several recent experiments and theoretical calculations. Considering that we estimate that our error bars have a better than $90 \%$ probability of encompassing the correct $D_{0}$ value, we question the recent upper bound inferred from Stark anticrossing spectroscopy.

\section{Acknowledgement:}

Part of this work was performed under a grant of computer time provided by the NAS Facility. P. R. Taylor was supported by NASA grant NCC 2-371. We would like to thank E. A. Carter, W. C. Lineberger, and G. A. Petersson for communicating results in advance of publication. 


\section{REFERENCES}

1. P. G. Green, J. L. Kinsey, and R. W. Field, J. Chem. Phys. 91 (1989) 5160.

2. J. Segall, R. Lavi, Y. Wen, and C. Wittig, J. Phys. Chem. 93 (1989) 7287.

3. L. A. Curtiss and J. A. Pople, J. Chem. Phys. 91 (1989) 2420.

4. J. A. Montogmery and G. A. Petersson, Chem. Phys. Letters, in press.

5. K. M. Ervin, S. Gronert, S. E. Barlow, M. K. Gilles, A. G. Harrison, V. M. Bierbaum, C. H. DePuy, W. C. Lineberger, and G. B. Ellison, J. Am. Chem. Soc. (submitted).

6. M. R. A. Blomberg and P. E. M. Siegbahn, J. Chem. Phys. 78 (1983) 5682. See also S. R. Langhoff and E. R. Davidson, Int. J. Quantum Chem. 8 (1974) 61.

7. R. J. Gdanitz and R. Ahlrichs, Chem. Phys. Letters 143 (1988) 413.

8. R. Ahlrichs, P. Scharf, and C. Ehrhardt, J. Chem. Phys. 82 (1985) 890.

9. D. P. Chong and S. R. Langhoff, J. Chem. Phys. 84 (1986) 5606.

10. J. Almlöf and P. R. Taylor, J. Chem. Phys. 86 (1987) 4070.

11. F. B. van Duijneveldt, IBM Research Report No. RJ 945 (1971).

12. C. W. Bauschlicher and S. R. Langhoff, Chem. Phys. Letters 135 (1987) 67.

13. C. W. Bauschlicher, S. R. Langhoff, and P. R. Taylor, J. Chem. Phys. 88 (1988) 2540.

14. MOLECULE-SWEDEN is an electronic structure program system written by J. Almlöf, C. W. Bauschlicher, M. R. A. Blomberg, D. P. Chong, A. Heiberg, S. R. Langhoff, P.-A. Malmqvist, A. P. Rendell, B. O. Roos, P. E. M. Siegbahn, and P. R. Taylor.

15. J. A. Pople, M. Head-Gordon, D. J. Fox, K. Raghavachari, and L. A. Curtiss, J. Chem. Phys. 90 (1989) 5622.

16. J. A. Pople, M. Head-Gordon, and K. Raghavachari, J. Chem. Phys. 87 (1987) 5968.

17. C. W. Bauschlicher, S. R. Langhoff and P. R. Taylor, Adv. Chem. Phys. (in press).

18. G. Strey and I. M. Mills, J. Mol. Struct. 59 (1976) 103.

19. H. Kanamori and E. Hirota, J. Chem. Phys. 89 (1988) 3962.

20. H. Kanamori, K. Seki, and E. Hirota, J. Chem. Phys. 87 (1987) 73. 
21. M. E. Jacox, Chem. Phys. 7 (1975) 424.

22. M. Peric, S. D. Peyerimhoff, and R. J. Buenker, paper communicated to the authors by R. F. Curl.

23. A. M. Wodtke and Y. T. Lee, J. Phys. Chem. 89 (1985) 4744.

24. H. Shiromaru, Y. Achiba, K. Kimura, and Y. T. Lee, J. Phys. Chem. 91 (1987) 17.

25. C. J. Wu and E. A. Carter, J. Am. Chem. Soc, submitted for publication.

26. P. M. W. Gill, J. A. Pople, L. Radom, and R. H. Nobes, J. Chem. Phys. 89 (1988) 7307. 
Table I. Basis set calibration studies of $D_{e}$ at the CPF level.

\begin{tabular}{|c|c|c|c|}
\hline \multirow[t]{2}{*}{ Basis set } & \multicolumn{2}{|c|}{$\operatorname{Energy}\left(E_{h}\right)$} & \multirow[t]{2}{*}{$D_{e}(\mathrm{kcal} / \mathrm{mole})^{a}$} \\
\hline & Molecule ${ }^{b}$ & Fragment $^{c}$ & \\
\hline$\left[\begin{array}{llllll}4 s & 3 p & 2 d & 1 f / 3 s & 2 p & 1 d\end{array}\right]$ & -77.183339 & -76.962320 & 138.7 \\
\hline$\left[\begin{array}{llllll}4 s & 3 p & 2 d & 1 f / 4 s & 2 p & 1 d\end{array}\right]$ & -77.183994 & -76.963765 & 138.2 \\
\hline$\left[\begin{array}{llllll}5 s & 4 p & 3 d & 2 f / 4 s & 3 p & 2 d\end{array}\right]$ & -77.191595 & -76.971225 & 138.3 \\
\hline$\left[\begin{array}{lllllllll}5 s & 4 p & 3 d & 2 f & 1 g / 4 s & 3 p & 2 d\end{array}\right]$ & -77.197034 & -76.976115 & 138.6 \\
\hline
\end{tabular}

a The MCPF $D_{e}$ values are uniformly $0.3 \mathrm{kcal} / \mathrm{mole}$ smaller.

${ }^{b} \mathrm{R}(\mathrm{C}-\mathrm{C})=2.292$ and $\mathrm{R}(\mathrm{C}-\mathrm{H})=2.010 a_{0}$.

${ }^{c} \mathrm{R}(\mathrm{C}-\mathrm{C})=2.308$ and $\mathrm{R}(\mathrm{C}-\mathrm{H})=2.016 a_{0}$. 


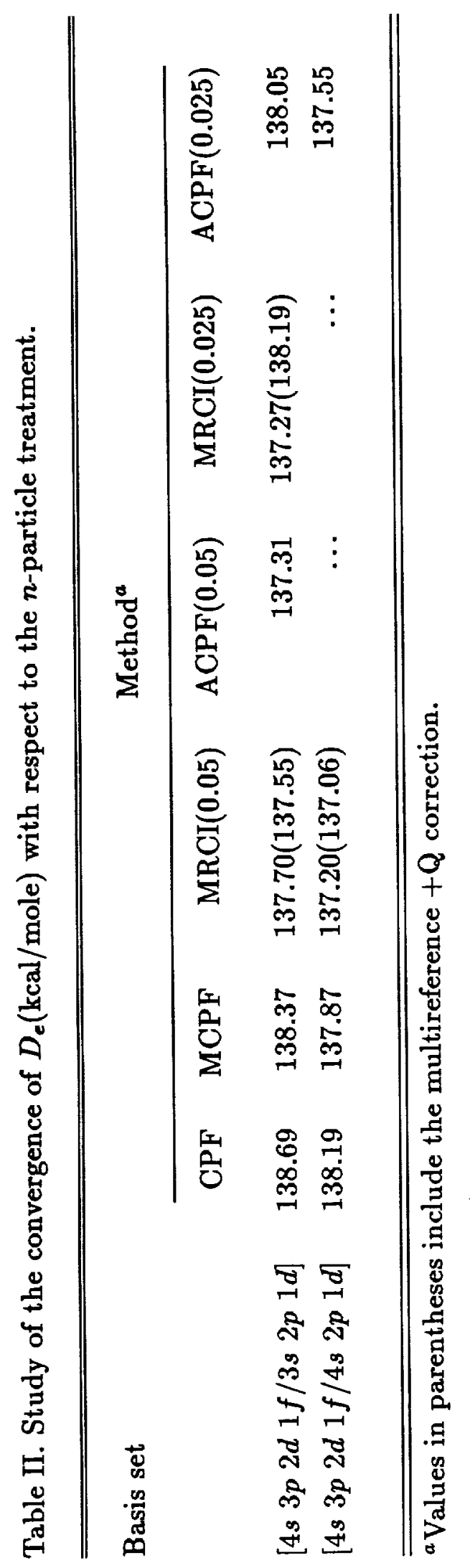


Table III. CPF harmonic frequencies $\left(\mathrm{cm}^{-1}\right)$ for $\mathrm{C}_{2} \mathrm{H}_{2}$ and $\mathrm{C}_{2} \mathrm{H}$.

Mode

Harmonic frequencies

Theory

Experiment ${ }^{a}$

A. $\mathrm{C}_{2} \mathrm{H}_{2}$

$\sigma_{g}(\mathrm{CH}$ stretch)

3544

3497

$\sigma_{g}(\mathrm{CC}$ stretch $)$

2034

2011

$\sigma_{u}(\mathrm{CH}$ stretch)

3444

3415

$\pi_{g}$ (bend)

616

624

$\pi_{u}$ (bend)

761

747

B. $\mathrm{C}_{2} \mathrm{H}$

$\nu_{1}$ (CC stretch)

2017

$1840.6^{b}$

$\nu_{2}$ (bend)

275

$371.6^{c}$

$\nu_{3}$ (CH stretch)

3328

${ }^{a}$ Ref. 18.

${ }^{b}$ Ref. 20.

${ }^{c}$ Ref. 19. 
Table IV. Summary of recent experimental and theoretical $D_{0}$ values $(\mathrm{kcal} / \mathrm{mole})$ for acetylene.

\begin{tabular}{llr}
\hline$D_{0}(\mathrm{HCC}-\mathrm{H})$ & Method \\
& \multicolumn{1}{c}{$\quad$ Refperimental } \\
& & \\
$\leq 126.647 \pm 0.002$ & Stark anti-crossing spectroscopy & 1 \\
$127 . \pm 1.5$ & HCCH $+h \nu \rightarrow \mathrm{C}_{2} \mathrm{H}+\mathrm{H}(\mathrm{K} . \mathrm{E})$. & 2 \\
$131.3 \pm 0.7$ & $\Delta \mathrm{H}_{\text {acid }}(\mathrm{HCCH})+\mathrm{EA}\left(\mathrm{C}_{2} \mathrm{H}\right)-\mathrm{IP}(\mathrm{H})$ & 5 \\
$132 . \pm 2$ & HCCH $+h \nu \rightarrow \mathrm{C}_{2} \mathrm{H}(\mathrm{K} . \mathrm{E})+.\mathrm{H}$ & 23 \\
$132.6 \pm 1.2$ & photoionization of $\mathrm{HCCH}$ & 24
\end{tabular}

Theoretical

$133.5 \pm 2.3$

$131.54 \pm 0.45$

129.7

$130.1 \pm 1.0$
G1

QCI

MRCI

MRCI
1

2

5

23

24

3

4

25

This work 